\title{
Whole blood neutrophil chemiluminescence in children with diabetes depending on their clinical condition
}

\author{
MAGDA KACZMAREK ${ }^{1}$, ALEKSANDRA LEWANDOWICZ-USZYŃSKA², ZDZISEAWA IWANICKA ${ }^{3}$, \\ ADAM JANKOWSKI
}

${ }^{1}$ Student Scientific Society at then $3^{\text {rd }}$ Department and Clinic of Pediatrics, Immunology and Rheumatology of Developmental Age, Wroclaw Medical University

${ }^{2} 3^{\text {rd }}$ Department and Clinic of Pediatrics, Immunology and Rheumatology of Developmental Age, Wroclaw Medical University ${ }^{3}$ Department and Clinic of Endocrinology, Wroclaw Medical University

\begin{abstract}
The study deals with the examination of the metabolic activity of neutrophils tested with spontaneous and stimulated (fMLP, PMA, Zymosan) chemiluminescence $(C L)$ in children with type 1 diabetes depending on their clinical conditions. We examined 87 children with type 1 diabetes from 4 to 21 years (52 girls and 35 boys, mean age - 13 years). Due to their clinical conditions, the patients were divided into three subgroups: patients in good condition (25 children), patients in mediocre condition (54 children), and patients in severe condition (8 children). The control group consisted of 23 persons - healthy volunteers from 20 to 25 years old (mean age - 23 years). We estimated the maximum levels of chemiluminescence (CLmax) with a luminometer (Microlumat LB 96P of E\&G Barthold), the results were given in RLU/s. We found decreased oxide metabolism of neutrophils in patients with diabetes in all clinical conditions, both spontaneous and stimulated compared to the control group. The lowest values of neutrophil chemiluminescence were in children in mediocre condition.
\end{abstract}

Key words: diabetes, clinical condition, neutrophil chemiluminescence, children.

(Centr Eur J Immunol 2012; 37 (4): 345-349)

\section{Introduction}

Type 1 diabetes develops most often in children and adolescents. Before it manifests itself, a complex process of pancreatic islets degradation develops within the pancreatic islets where both genetic and immunological factors are of crucial significance [1,2]. Both cell-mediated and humoral mechanisms are involved in the destruction of $\beta$ cells of the islets of Langerhans. This leads to autoimmune reaction as a sign of breaking tolerance to autoantigens and initiation of an immune response against one's own tissues [3, 4]. Diabetes may manifest itself many months before the process. The direct way which leads to the selective degradation of $\beta$ cells in IDDM (insulin dependent diabetes) is still poorly understood. An important role in the destruction process is attributed to pro- inflammatory cytokines, especially interleukin 1 (IL-1), and nitric oxide and oxygen free radicals (RT) [4-7]. Interleukin 1 released by monocytes and macrophages and also by Langerhans cells and vascular endothelium inhibits the synthesis and release of insulin, resulting in a long-term exposure to permanent damage of $\beta$ cells $[8,9]$. This cytokine activates immune cells to release TNF- $\alpha$ and IFN- $\gamma$ which then can induce neutrophils and macrophages to generate RT [10, 11]. Getting into the extracellular space, RT released from the cells damage surrounding cells, contributing to the expansion of tissue destruction and the spread of infection. The toxic activity of RT is boosted by low contents of enzymes in the cells of the islets of Langerhans (MnSOD levels in them are small even after cytokine stimulation) which decompose these highly reactive agents. The state is

Correspondence: Aleksandra Lewandowicz-Uszyńska, MD, PhD, $3^{\text {rd }}$ Department and Clinic of Pediatrics, Immunology and Rheumatology of Developmental Age, Wroclaw Medical University, Koszarowa 5, 51-149 Wrocław, phone: +48 $713925396,+48713925397$, e-mail: alusz@dilnet.wroc.pl 
also enhanced by increased secretory activity of $\beta$ cells. Metabolically active cells are much more susceptible to the damaging effects of cytokines and RT [12]. The participation of RT in the etiopathogenesis of IDDM is also confirmed by the results of tests with the use of "RT scavengers" [13]. Antioxidant compounds protect pancreatic cells from the toxic effects of cytokines [IL-1, interferon $\gamma$ (IFN- $\gamma$ ), tumor necrosis factor (TNF)] and alloxan, while administered to animals with genetically induced diabetes retard its development $[14,15]$.

The aim of this study is to assess spontaneous and stimulated metabolic activity of neutrophils in the whole blood in children with type 1 diabetes depending on their clinical conditions.

\section{Material and methods}

The study included 87 children and adolescents with type 1 diabetes at the age of 4 to 21 years (52 girls and 35 boys, mean age -13 years) treated at the Clinic of Developmental Age Endocrinology, Wroclaw Medical University. All patients received humanized insulin administered repeatedly throughout 24 hours. Information about the current course of the disease was obtained from interviews and medical records. Due to the clinical condition and degree of metabolic control of diabetes, patients were divided into three subgroups:

- good condition - 25 children (in whom acidosis occurred only once in the course of diabetes, and at the time of the study, diabetes was metabolically controlled, $\mathrm{HbA}_{1 \mathrm{C}}$ $<6.4 \%$, cholesterol and triglycerides - correct values);

- mediocre condition - 54 children (acidosis occurred 2-3 times in the course of the disease, $\mathrm{HbA}_{1 \mathrm{C}} 6.5-7.5 \%$, without glycemia and glycosuria);

- severe condition -8 children (patients exhibit characteristics of uncontrolled metabolic diabetes, $\mathrm{HbA}_{1 \mathrm{C}}>7.5 \%$, frequent hyperglycemia and glycosuria, hyperlipidemia were observed; the control group consisted of 23 people - healthy volunteers aged 20 to 25 years (mean age -23 years); they were students, because it is very difficult to obtain healthy children's blood for testing.

The study was approved by the Bioethics Committee (858/01-03) at Wroclaw Medical University.

The material for this study was venous blood, collected into plastic tubes with heparin in a closed system, while performing basic diagnostic tests or checks. The metabolic activity of neutrophils was determined with a luminoldependent chemiluminescence test $[16,17]$. Spontaneous and stimulated neutrophil CL [fMLP, opsonized Zymosan, phorbol 12-myristate 13-acetate (PMA)] was studied in 96-well, white microplate (LB96P-WMP) according to Lewkowicz et al. [18]. The order of addition and volume of reagents are shown in Table 1.

Each of the systems was repeated three times, and the arithmetic mean of the values received was calculated. The chemiluminescence was tested with a luminometer (MicroLumat LB 96P of E\&G BARTHOLD). The device was controlled by WinGlow software in the Windows environment. Spot measurements were taken within 0.2 seconds every 60 seconds at $37^{\circ} \mathrm{C}$. The test lasted 45 minutes. Results are expressed in RLU (Relative Light Units/s) as the maximum chemiluminescence $\left(\mathrm{CL}_{\max }\right)$. Since $\mathrm{CL}$ depends linearly on the number of neutrophils, while heme compounds reduce its values, the chemiluminescence results were adjusted to their absolute number and haemoglobin content in the blood tested by the formula:

$\mathrm{Hb}[\%]$

$\overline{\mathrm{PMN}[\%] \times \mathrm{WBC} \text { [thous./100 } \mu \mathrm{l}]}$

$\mathrm{CL}_{\text {CALCULATED }}=\mathrm{CL}_{\text {DETERMINED }} \mathrm{Xy}$

where $\mathrm{Hb}$ is haemoglobin, and PMN is the percentage of neutrophils in the total number of white blood cells - WBC.

A statistical analysis conducted using the Mann-Whitney $U$ test involved designation of the arithmetic mean, standard deviation, and the level of significance $(p<0.05)$ for each parameter of neutrophil CL in examined patients and the control group. Using the Kruskal-Wallis ANOVA by Ranks test, box and whisker plots were made showing individual parameters of neutrophil CL including the mean, standard deviation and standard error for examined patients and the control group.

\section{Results}

Significantly lower levels of spontaneous neutrophil CL were shown in all children with diabetes (regardless of their clinical condition) compared to the control group (Fig. 1).

Table 1. The order of addition and volume of reagents

\begin{tabular}{llll}
\hline No stimulation & FMLP & PMA & Zymosan \\
\hline 1. PBS $40 \mu 1$ & 1. PBS $20 \mu 1$ & 1. PBS $20 \mu 1$ & 1. PBS 20 $\mu 1$ \\
\hline 2. Luminol $20 \mu 1$ & 2. fMLP $20 \mu 1$ & 2. PMA $20 \mu 1$ & 2. Zymosan 20 $\mu 1$ \\
\hline 3. Whole blood $40 \mu 1$ & 3. Luminol $20 \mu 1$ & 3. Luminol $20 \mu 1$ & 3. Luminol 20 $\mu 1$ \\
\hline & 4. Whole blood $40 \mu 1$ & 4. Whole blood $40 \mu 1$ & 4. Whole blood $40 \mu 1$ \\
\hline
\end{tabular}




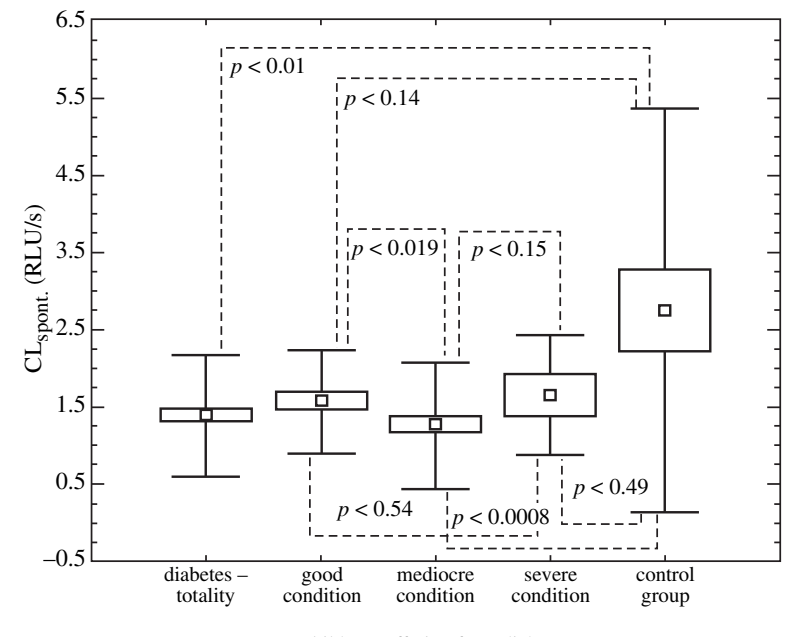

children suffering from diabetes

$$
\begin{aligned}
& \text { I Standard deviation } \\
& \square \text { Standard error }
\end{aligned}
$$

Fig. 1. Spontaneous chemiluminescence of neutrophils in children with diabetes compared to the control group

There were no statistically significant differences in spontaneous neutrophil CL in children in good and severe condition compared to the control group. There were statistically significant lower levels of spontaneous neutrophil CL in children in mediocre condition as compared to the control group $(p<0.0008)$, and compared to children in good condition $(p<0.019)$. The results of $\mathrm{CL}_{\max }$ after stimulation with $\mathrm{AMLP}$ in the examined groups are presented in Fig. 2. There were significantly lower levels of $\mathrm{CL}_{\max }$ in children with diabetes (all clinical conditions together) and children in mediocre condition as compared to the control group. There were no statistically significant differences in the $\mathrm{CL}_{\text {max }}$ levels between children in good and severe conditions, and in comparison to the control group. The results of $\mathrm{CL}_{\max }$ after stimulation with PMA in the examined groups are shown in Fig. 3. All subgroups of children with diabetes demonstrated significantly lower $\mathrm{CL}_{\max }$ levels compared to the control group, while the lowest values were found in children in mediocre condition. The opsonized Zymosan stimulation of neutrophils showed significantly lower $\mathrm{CL}_{\max }$ values in all examined subgroups of children with diabetes. As in the case of fMLP and PMA stimulation, the lowest neutrophil CL levels were in children in mediocre condition.

\section{Discussion}

Diabetes is one of diseases the development of which is affected by the presence of RT. As a result of oxidative stress, elevated levels of lipid peroxidation products such as MDA, reduced level of antioxidants in the body fluids

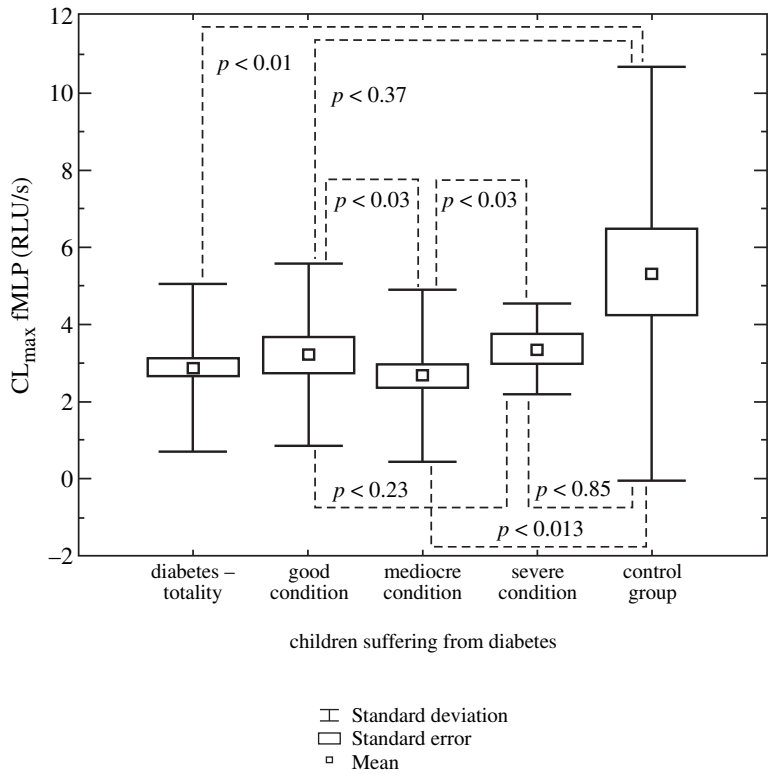

Fig. 2. The maximum value of neutrophil chemiluminescence after fMLP stimulation in children with diabetes compared to the control group

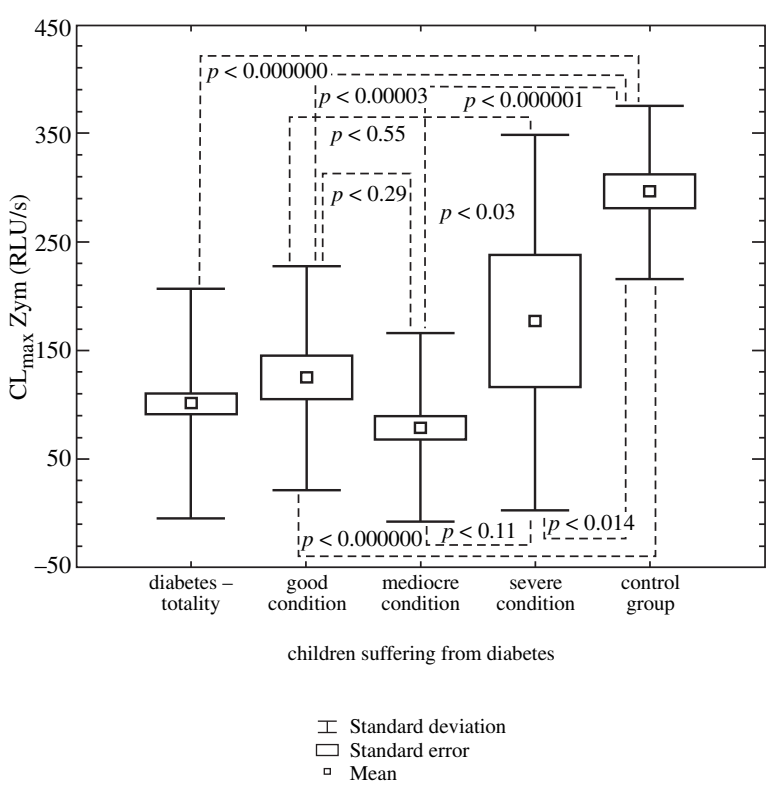

Fig. 3. The maximum value of neutrophil chemiluminescence after opsonized Zymosan stimulation in children with diabetes compared to the control group

and within cells are observed in diabetes [19]. In the early stage of type 1 diabetes in patients at developmental age, abnormalities are observed in the oxidation-reduction system, which is supported by studies conducted by Telci $e t$ al. [20]. They found elevated levels of markers of oxidative damage to proteins and decreased antioxidant capacity of 
plasma in children with type 1 diabetes compared to the control group. In the present study we observed a decrease in neutrophil oxidative metabolism in children with diabetes compared to healthy people. We also observed impaired neutrophil response to stimulating factors, as well as a decrease of CL in the system without a stimulator (spontaneous CL) which may prove a functional "exhaustion" or "fatigue" of the cells. Studies conducted by Zgliczyński et al. [21] show that neutrophils may be present in different physiological states. The following neutrophils were distinguished: resting, pre-activated, activated and exhausted. Pre-activated neutrophils are in the state of functional readiness and the activation of the next stimulus causes their stronger response such as increased production of free radicals. Activated cells show a multiple increase in the release of RT [22], and are characteristic of inflammation, extensive burns and metastatic cancer [23, 24]. In diabetes, hyperglycemia may stimulate granulocytes, as in the sorbitol pathway with an increase in DAG synthesis, it activates protein kinase $\mathrm{C}$ - an enzyme crucial to many changes taking place in a cell [neutrophil stimulants may also be cytokines IL-1, IL-6, IL-8, TNF- $\alpha$, glycated proteins (AGE)]. Their level increases in the blood of people with uncontrolled diabetes $[25,26]$. Chronic neutrophil pre-activation in a high concentration of glucose in the body may lead to a decrease in the sensitivity of these cells to subsequent stimuli. This can be observed in only a slight increase in oxidative metabolism of neutrophils in response to stimuli (fMLP, PMA, Zymosan) in children with type 1 diabetes in comparison with healthy persons (the control group). Functional exhaustion of cells which results in low levels of spontaneous and stimulated chemiluminescence was also observed in other diseases such as in children or adults with recurrent respiratory tract infections [27-29]. Impaired metabolic activity of neutrophils may be caused by various factors: long-term stress, medications (antibiotics), environmental pollution, it may also be genetic. Impaired synthesis of RT by neutrophils affects the body's defence against pathogenic microorganisms, and thus its tendency to infections. It has been observed that people with diabetes are more susceptible to bacterial infection, as well as fungal and viral infections [30]. It is assumed that defects in neutrophil oxidative metabolism in children with type 1 diabetes are related, inter alia, to elevated blood glucose. In all systems tested (spontaneous CL, after stimulation with fMLP, PMA, Zymosan) significantly higher neutrophil chemiluminescence in children in good clinical condition compared to children in overall mediocre condition was reported. Slightly higher $\mathrm{CL}_{\max }$ values were observed in children in serious condition, which disturbs the relationship. This could be due to the too small number of people in the selected group $(n=8)$ compared to children in good clinical condition $(n=25)$ and mediocre clinical condition $(n=54)$. It is worth emphasizing that all the three subgroups whose clinical conditions were taken into account, included children with both short- and long-term diabetes, which significantly may affect the results. The presence of comorbidities, medications, blood glucose levels also affect the final results obtained. Gallacher et al. [31] who conducted studies on people with type 1 and type 2 diabetes, also obtained lower CL values compared to the control group. These authors also showed a negative correlation between chemiluminescence and $\mathrm{HbA}_{1 \mathrm{C}}$ levels in the blood. Therefore, weakening neutrophil bactericidal function should be associated not only with direct changes in blood glucose levels, but also with poor metabolic control. Another cause of impaired neutrophil oxidative metabolism may be response of these cells to insulin. Studies conducted in vitro by Oldenborg et al. [32] on healthy people showed inhibition of neutrophil chemiluminescence after stimulation with fMLP by insulin. Contrasting results were obtained by Scatena et al. [33] who measured opsonized Zymosan-stimulated neutrophil CL in people with diabetes, showing increased CL in comparison to the control group. Saeed et al. [34] showed impaired neutrophil oxidative metabolism using CL test in patients with diabetes, and they attributed the inhibitory role to acidic metabolites (acetoacetate) present in the serum of these patients. Delamaire et al. showed impaired neutrophil oxidative metabolism in patients with type 1 diabetes [35]. Phorbol 12-myristate 13-acetate and Zymosan-induced CL of neutrophils was lower compared to healthy people. Although a lot of studies report the participation of free radicals in the pathology of diabetes and complications typical of this disease, the present study failed to capture the moment of severe oxidative burst measured with a chemiluminescence test. Perhaps the state took place before the onset of clinical symptoms suggestive of the diagnosis of diabetes. The results of the study show decreased neutrophil oxygen metabolism in all test systems, which indicates their exhaustion. A tendency to the occurrence of recurrent infections is often observed in patients suffering from diabetes. On the one hand, the predisposing factors may include glycemic fluctuations and the resulting metabolic disorders, and, on the other hand, abnormal oxidative metabolism of neutrophils may explain the cause of the greater susceptibility of diabetic patients to infection.

\section{The authors declare no conflict of interest.}

\section{References}

1. Goh SY, Cooper ME (2008): The role of advanced glycation end products in progression and complications of diabetes. J Clin Endocrinol Metab 93: 1143-1152.

2. Nerup J, Mandrup-Poulsen T, Helqvist S, et al. (1994): On the pathogenesis of IDDM. Diabetologia 37: S82-S89.

3. Knip M, Siljander H (2008): Autoimmune mechanisms in type 1 diabetes. Autoimmun Rev 7: 550-557.

4. Kantárová D, Buc M, Stuchlíková M, et al. (2006): Etiopathogenesis of autoimmune diabetes mellitus in human. A review. Centr Eur J Immunol 31: 102-110. 
5. Kolb H, Kolb-Bachofen V (1992): Nitric oxide: a pathogenetic factor in autoimmunity. Immunol Today 13: 157-160.

6. Iwanicka Z, Lewandowicz-Uszyńska A, Głab E, Kotschy B (2006): Relationship between nitrogen oxide and the degree of metabolic control of diabetes mellitus type 1 in children and adolescents. Wiad Lek 59: 27-31.

7. Alba-Loureiro TC, Munhoz CD, Martins JO, et al. (2007): Neutrophil function and metabolism in individuals with diabetes mellitus. Braz J Med Biol Res 40: 1037-1044.

8. Komosińska K, Olczyk K (1995): The role of free radicals in pathogenesis of insulin dependent diabetes mellitus. Postepy Hig Med Dosw 49: 733-746.

9. Mandrup-Poulsen T, Corbett JA, McDaniel ML, Nerup J (1993): What are types and cellular sources of free radicals in the pathogenesis of type1 (insulin-dependent) diabetes mellitus? Diabetologia 36: 470-471.

10. Hatanaka E, Monteagudo PT, Marrocos MS, Campa A (2006): Neutrophils and monocytes as potentially important sources of proinflammatory cytokines in diabetes. Clin Exp Immunol 146: 443-447.

11. Rabinovitch A, Suarez WL, Thomas PD, et al. (1992): Cytotoxic effects of cytokines on rat islets: evidence for involvement of free radicals and lipid peroxidation. Diabetologia 35: 409-413.

12. Maritim AC, Sanders RA, Watkins JB 3rd (2003): Diabetes, oxidative stress, and antioxidants: a review. J Biochem Mol Toxicol 17: 24-38.

13. Rabinovitch A, Sumoski W, Rajotte RV, Warnock GL (1990): Cytotoxic effects of cytokines on human pancreatic islet cells in monolayer culture. J Clin Endocrinol Metab 71: 152-156.

14. Pinzani P, Petruzzi E, Orlando C, et al. (1998): Serum antioxidant capacity in healthy and diabetic subjects as determined by enhanced chemiluminescence. J Biolumin Chemilumin 13: 321-325.

15. Terekhina NA, Khorobrykh OIu, Khorobrykh TP (1998): Antioxidant enzyme activity in the erythrocytes of rats with alloxan diabetes. Patol Fiziol Eksp Ter 4: 25-26.

16. Allen RC (1986): Phagocytic leukocyte oxygenation activities and chemiluminescence: a kinetic approach to analysis. Methods Enzymol 133: 449-493.

17. Lewandowicz-Uszyńska A, Jargulińska-Forysiak E (2003): Application of neutrophils chemiluminescence test in medical diagnostics. Centr Eur J Immunol 28: 131-137.

18. Lewkowicz P, Lauk-Pachuła B, Górańska N, et al. (1999): The attempt of standardization of the whole blood chemiluminescence assessment as a method of examining the human granulocytes' function in the "in vitro" research. Diagn Lab 35: 497-510.

19. Gil-del Valle L, de la C Milian L, Toledo A, et al. (2005): Altered redox status in patients with diabetes mellitus type I. Pharmacol Res 51: 375-380.

20. Telci A, Cakatay U, Salman S, et al. (2000): Oxidative protein damage in early stage type 1 diabetic patients. Diabetes Res Clin Pract 50: 213-223.

21. Zgliczyński JM, Kwasnowska E, Stelmaszyńska T, et al. (1988): Functional states of neutrophils as suggested by whole blood chemiluminescence. Acta Biochim Pol 35: 331-342.

22. Paśnik J (1998): TNF-alpha preactivation (priming) of neutrophils - effect on selected neutrophil functions. Post Hig Med Dosw 52: 139-155.

23. Favier A (2006): Oxidative stress in human diseases. Ann Pharm Fr 64: 390-396.
24. Valko M, Leibfritz D, Moncol J, et al. (2007): Free radicals and antioxidants in normal physiological functions and human disease. Int J Biochem Cell Biol 39: 44-84.

25. Hunt JV, Dean RT, Wolff SP (1988): Hydroxyl radical production and autoxidative glycosylation. Glucose autoxidation as the cause of protein damage in the experimental glycation model of diabetes mellitus and ageing. Biochem J 256: 205212.

26. Erciyas F, Taneli F, Arslan B, Uslu Y, et al. (2004): Glycemic control, oxidative stress, and lipid profile in children with type 1 diabetes mellitus. Arch Med Res 35: 134-140.

27. Lewandowicz-Uszyńska A, Jankowski A (2004): Chemiluminescence of neutrophiles stimulated by opsonized Zymosan in children with asthma bronchiale and pneumonia. Proc SPIE 5566: 165-170.

28. Lewandowicz-Uszyńska A, Jankowski A (2001): Application of neutrofils chemiluminescence test in the differential diagnosis of asthma and rrti the remission period in children. Proc SPIE 91: 4515.

29. Zabuska-Jabłońska K, Broniek A (1997): Disturbances of polymorphonuclear neutrophil leukocyte chemiluminescence in patients with chronic or recurrent respiratory tract infections. Pneumonol Alergol Pol 65: 649-656.

30. Zozulińska D, Wierusz-Wysocka B (2000): Wybrane mechanizmy patogenetyczne przewlekłych powikłań cukrzycy. II. Rola granulocytów obojętnochłonnych. Diabetol Pol 7: 198.

31. Gallacher SJ, Thomson G, Fraser WD, et al. (1995): Neutrophils bactericidal function in diabetes mellitus: evidence for association with blood glucose control. Diabet Med 12: 916-920.

32. Oldenborg PA (1999): Effects of insulin on N-formylmethionyl-leucyl-phenylalanine (fMet-Leu-Phe)-stimulated production of reactive oxygen metabolites from normal human neutrophils. Inflamm Res 48: 404-411.

33. Scatena R, Nocca G, De Sole P, et al. (1998): Impaired reactive oxygen metabolism of phagocytic leukocytes in NIDDM patients. A role for non-enzymatic glycosylation of collagen. J Biolumin Chemilumin 13: 273-278.

34. Saeed FA, Castle GE (1998): Neutrophil chemiluminescence during phagocytosis is inhibited by abnormally elevated levels of acetoacetate: implications for diabetic susceptibility to infections. Clin Diagn Lab Immunol 5: 740-743.

35. Delamaire M, Maugendre D, Moreno M, et al. (1997): Impaired leucocyte functions in diabetic patients. Diabet Med 14: $29-34$ 